\section{Analysis and Synthesis of Markov Jump Linear Systems With Time-Varying Delays and Partially Known Transition Probabilities}

\author{
Lixian Zhang, El-Kébir Boukas, and James Lam
}

\begin{abstract}
In this note, the stability analysis and stabilization problems for a class of discrete-time Markov jump linear systems with partially known transition probabilities and time-varying delays are investigated. The time-delay is considered to be time-varying and has a lower and upper bounds. The transition probabilities of the mode jumps are considered to be partially known, which relax the traditional assumption in Markov jump systems that all of them must be completely known a priori. Following the recent study on the class of systems, a monotonicity is further observed in concern of the conservatism of obtaining the maximal delay range due to the unknown elements in the transition probability matrix. Sufficient conditions for stochastic stability of the underlying systems are derived via the linear matrix inequality (LMI) formulation, and the design of the stabilizing controller is further given. A numerical example is used to illustrate the developed theory.
\end{abstract}

Index Terms-Linear matrix inequality (LMI), Markov jump linear systems, stochastic stability and stabilization, time-varying delays, transition probabilities.

\section{INTRODUCTION}

The past decades have witnessed extensive research on time-delay systems, and many analysis and synthesis results using delay-dependent approach have been widely reported in concern of conservatism, see for example, [1]-[5]. Very recently, a new so-called delay-range-dependent concept was proposed and much less conservative stability criteria were developed by constructing more appropriate Lyapunov functional for continuous-time case and discrete-time case [6], [7], respectively. The time-varying delays are considered to vary in a range and thereby more applicable in practice.

On the other hand, Markov jump systems with or without time delays have also attracted much attention due to their widely practical applications in manufacturing systems, power systems, aerospace systems and networked control system, etc [8], [9]. In such systems, the transition probabilities of the jumping process are crucial and so far, almost all the issues on Markov jump system have been investigated assuming the complete knowledge of these transition probabilities. However, the likelihood to obtain the complete knowledge on the transition probabilities is questionable and the cost is probably high. Take VTOL (vertical take-off landing) helicopter system in the aerospace industry for example, the airspeeds variation involved in the system matrices are modeled as a Markov Chain [10]. However, not all the probabilities of the jumps among multiple airspeeds are easy to measure. In fact, from 135 knots (normal value) to 135 knots (dwell in one mode), one

Manuscript received December 04, 2007; revised April 04, 2008 and May 23, 2008. Current version published November 05, 2008. This work was supported by NSERC-Canada Grant OPG0036444 and RGC HKU 7031/07P. Recommended by Associate Editor M. Xiao.

L. Zhang is with the Space Control and Inertial Technology Research Center, Harbin Institute of Technology, Harbin 150001, China (e-mail: lixianzhang@hit.edu.cn).

E.-K. Boukas is with the Department of Mechanical Engineering, Ecole Polytechnique de Montreal, Montreal, QC H3C 3A7 Canada.

J. Lam is with the Department of Mechanical Engineering, University of Hong Kong, Hong Kong, China.

Color versions of one or more of the figures in this paper are available online at http://ieeexplore.ieee.org.

Digital Object Identifier 10.1109/TAC.2008.2007867 may obtain the accurate probability or estimate a scope (uncertain one) effortlessly, but for the cases from 135 knots to any of 60,70 or 80 knots, for instance, the probability is likely not accurate and the uncertainties bounds for them are quite ideal. The same problems may arise in other practical systems with Markovian jumps. Thus, it is significant and challenging to further study more general jump systems with partially known transition probabilities from control perspectives, especially with time-varying delays included. More recently, some attention have been already drawn to the class of systems without time delays for both continuous-time and discrete-time [11]. However, what is the exact impact of the unknown transition probabilities to the system performance, say, to the maximal delay bounds (or ranges) if the systems are involved with time delay? As expected, a compromise between the complexity of obtaining all the transition probabilities and system performance benefits (the maximal accessible delay range in this note) should be reached as required in practice. Note that the time-varying delays will cover the mode-dependent delays in autonomous hybrid systems like, Markov jump systems, see [12] or arbitrary switching systems, see [4], since the mode variation will be finally time-driven in such systems.

In this note, we are interested in the stability analysis and stabilization synthesis problems for a class of discrete-time Markov jump linear systems (MJLS) with partially known transition probabilities and time-varying delays. The contribution of our note is twofold. Firstly, the proposed systems will be more general and cover the cases of systems with completely unknown or known transition probabilities. Secondly, an advancement of the delay-range-dependent concept is introduced here and naturally, less conservative stability and stabilization conditions for the underlying systems will be obtained. The rest of the note is organized as follows. Section II gives the problem description and Section III establishes the delay-range-dependent stability for the systems with completely known transition probabilities which is further extended to obtain results for the systems with partially known transition probabilities. Section IV presents an illustrative example and Section $\mathrm{V}$ gives the conclusion.

Notation: The notation used in this note is standard. The superscript " $T$ " stands for matrix transposition, $\mathbb{R}^{n}$ denotes the $n$ dimensional Euclidean space; $\mathbb{N}^{+}$represents the sets of positive integers, respectively. $E[\cdot]$ stands for the mathematical expectation. In addition, in symmetric block matrices or long matrix expressions, we use ${ }^{a s t}$ as an ellipsis for the terms that are induced by symmetry and $\operatorname{diag}\{\cdots\}$ stands for a block-diagonal matrix. Matrices, if their dimensions are not explicitly stated, are assumed to be compatible for algebraic operations. The notation $P>0(\geq 0)$ means that $P$ is a real symmetric positive (semi-positive) definite matrix, and $M_{i}$ is adopted to denote $M(i)$ for brevity. $I$ and 0 represent, respectively, the identity matrix and zero matrix.

\section{PROBLEM Formulation}

Consider the following class of discrete-time Markov jump linear systems:

$$
\begin{aligned}
x(k+1) & =A\left(r_{k}\right) x(k)+B\left(r_{k}\right) u(k)+A_{d}\left(r_{k}\right) x(k-d(k)) \\
x(k) & =\phi(k), \quad k=-d_{M},-d_{M}+1, \ldots, 0
\end{aligned}
$$

where $x(k) \in \mathbb{R}^{n}$ is the state vector and $u(k)$ is the control input. The time-delay is considered to be time-varying and has a lower and upper bounds, $0<d_{m} \leq d(k) \leq d_{M}$, which is very common in practice.

The stochastic process $\left\{r_{k}, k \geq 0\right\}$ is described by a discrete-time homogeneous $\backslash$ Markov chain, which takes values in a finite set $\mathcal{I}=$ $\{1,2, \ldots, N\}$ with the following mode transition probabilities:

$$
\operatorname{Pr}\left(r_{k+1}=j \mid r_{k}=i\right)=\pi_{i j}
$$


where $\pi_{i j} \geq 0, \forall i, j \in \mathcal{I}$, and $\sum_{j=1}^{N} \pi_{i j}=1$. Furthermore, the transition probabilities matrix is defined by

$$
\pi=\left[\begin{array}{cccc}
\pi_{11} & \pi_{12} & \cdots & \pi_{1 N} \\
\pi_{21} & \pi_{22} & \cdots & \pi_{2 N} \\
& & \ddots & \\
\pi_{N 1} & \pi_{N 2} & \cdots & \pi_{N N}
\end{array}\right]
$$

For $r_{k}=i \in \mathcal{I}$, the system matrices of the $i$ th mode are denoted by $\left(A_{i}, B_{i}, A_{d i}\right)$, which are assumed known.

In addition, the transition probabilities of the Markov chain in this note are considered to be partially available, namely, some elements in matrix $\pi$ are time-invariant but unknown. For instance, a system (1) with four modes will have the transition probabilities matrix $\pi$ as

$$
\pi=\left[\begin{array}{cccc}
\pi_{11} & ? & \pi_{13} & ? \\
? & ? & ? & \pi_{24} \\
\pi_{31} & ? & \pi_{33} & ? \\
? & ? & \pi_{43} & \pi_{44}
\end{array}\right]
$$

where "?" represents the unavailable elements. For notation clarity, $\forall i \in \mathcal{I}$, we denote

$$
\mathcal{I}_{\mathcal{K}}^{i} \triangleq\left\{j: \text { if } \pi_{i j} \text { is known }\right\}, \quad \mathcal{I}_{\mathcal{U K}}^{i} \triangleq\left\{j: \text { if } \pi_{i j} \text { is unknown }\right\}
$$

Moreover, if $\mathcal{I}_{\mathcal{K}}^{i} \neq \emptyset$, it is further described as

$$
\mathcal{I}_{\mathcal{K}}^{i}=\left\{\mathcal{K}_{1}^{i}, \ldots, \mathcal{K}_{m}^{i}\right\}, \quad 1 \leq m \leq N
$$

where $\mathcal{K}_{m}^{i} \in \mathbb{N}^{+}$represents the $m$ th known element with the index $\mathcal{K}_{m}^{i}$ in the $i$ th row of matrix $\pi$. Also, we denote $\pi_{\mathcal{K}}^{i} \triangleq \sum_{j \in \mathcal{I}_{\mathcal{K}}^{i}} \pi_{i j}$ throughout the note.

Remark 1: Note that the transition probabilities in literature are commonly assumed to be completely available $\left(\mathcal{I}_{\mathcal{U}}^{i} \mathcal{K}=\emptyset, \mathcal{I}_{\mathcal{K}}^{i}=\mathcal{I}\right)$ or completely unavailable $\left(\mathcal{I}_{\mathcal{K}}^{i}=\emptyset, \mathcal{I}_{\mathcal{U} K}^{i}=\mathcal{I}\right)$. Moreover, in contrast with the uncertain transition probabilities studied recently, see for example, [13]-[15], no structure (polytopic ones), bounds (normbounded ones) or "nominal" terms (both) are required for the partially unknown elements in the transition probability matrix. Therefore, our transition probabilities considered here is more natural and reasonable to the Markov jump systems.

To describe the main objective of this note more precisely, let us now introduce the following definition for the underlying system.

Definition 1: [12] System (1) is said to be stochastically stable if for $u(k) \equiv 0$ and every initial condition $\phi(k) \in \mathbb{R}^{n}, k=-d_{M},-d_{M}+$ $1, \ldots, 0$ and $r_{0} \in \mathcal{I}$, the following holds,

$$
E\left\{\sum_{k=0}^{\infty}\|x(k)\|^{2} \mid \phi(\cdot), r_{0}\right\}<\infty
$$

The purposes of this note are to derive the stochastic stability criteria for system (1) when the transition probabilities are partially known, and to design a state-feedback stabilizing controller such that the resulting closed-loop system is stochastically stable. A mode-dependent controller is considered here with the form

$$
u(k)=K\left(r_{k}\right) x(k)
$$

where $K_{i}\left(r_{k}=i \in \mathcal{I}\right)$ is the controller gain to be determined.

\section{MAIN RESULTS}

In this section, we will first develop the stability criterion for the unforced system (1) (i.e., with $u(k) \equiv 0$ ) with completely known transition probabilities, and further give the stability conditions for the underlying systems with partially known transition probabilities and the corresponding controller design.

The following proposition gives the new stability criterion for system (1) with completely known transition probabilities, which is not only dependent on the delay upper bound $d_{M}$, but also the delay range $d_{r} \triangleq$ $d_{M}-d_{m}$.

Proposition 1: Consider the unforced system (1) with completely known transition probabilities (2). The corresponding system is stochastically stable if there exist matrices $P_{i}>0, i \in \mathcal{I}, Q>0$, $R>0, Z_{v}>0, v=1,2, M_{i v}, N_{i v}, S_{i v}, v=1,2,3, \forall i \in \mathcal{I}$ such that

$$
\left[\begin{array}{cccc}
-\overline{\mathcal{P}}_{i} & 0 & 0 & \Psi_{i 1} \\
* & -Z_{2} & 0 & \Psi_{i 2} \\
* & * & -Z_{1} & \Psi_{i 3} \\
* & * & * & \Psi_{i 4}
\end{array}\right]<0
$$

where

$$
\begin{aligned}
\Psi_{i 1} \triangleq & {\left[\begin{array}{llllll}
\overline{\mathcal{P}}_{i} A_{i} & \overline{\mathcal{P}}_{i} A_{d i} & 0 & 0 & 0 & 0
\end{array}\right] } \\
\Psi_{i 2} \triangleq & {\left[\begin{array}{llllll}
\sqrt{d_{M}} Z_{2}\left(A_{i}-I\right) & \sqrt{d_{M}} Z_{2} A_{d i} & 0 & 0 & 0 & 0
\end{array}\right] } \\
\Psi_{i 3} \triangleq & {\left[\begin{array}{lccccc}
\sqrt{d_{M}} Z_{1}\left(A_{i}-I\right) & \sqrt{d_{M}} Z_{1} A_{d i} & 0 & 0 & 0 & 0
\end{array}\right] } \\
\Psi_{i 4} \triangleq & {\left[\begin{array}{cccccc}
\Lambda_{i 11} & \Lambda_{i 12} & \Lambda_{i 13} & \sqrt{d_{M}} M_{i 1} & \sqrt{d_{r}} S_{i 1} & \sqrt{d_{M}} N_{i 1} \\
* & \Lambda_{i 22} & \Lambda_{i 23} & \sqrt{d_{M}} M_{i 2} & \sqrt{d_{r}} S_{i 2} & \sqrt{d_{M}} N_{i 2} \\
* & * & \Lambda_{i 33} & \sqrt{d_{M}} M_{i 3} & \sqrt{d_{r}} S_{i 3} & \sqrt{d_{M}} N_{i 3} \\
* & * & * & -Z_{1} & 0 & 0 \\
* & * & * & * & -Z_{1} & 0 \\
* & * & * & * & * & -Z_{2}
\end{array}\right] }
\end{aligned}
$$

with $\overline{\mathcal{P}}_{i} \triangleq \sum_{j \in \mathcal{I}} \pi_{i j} P_{j}$ and

$$
\begin{aligned}
& \Lambda_{i 11} \triangleq-P_{i}+\left(1+d_{r}\right) Q+R+M_{i 1}+N_{i 1}+M_{i 1}^{T}+N_{i 1}^{T} \\
& \Lambda_{i 12} \triangleq S_{1 i}-M_{i 1}+M_{i 2}^{T}+N_{i 2}^{T} \\
& \Lambda_{i 13} \triangleq-N_{1 i}-S_{i 1}+M_{i 3}^{T}+N_{i 3}^{T} \\
& \Lambda_{i 22} \triangleq-Q+S_{i 2}-M_{i 2}+S_{i 2}^{T}-M_{i 2}^{T} \\
& \Lambda_{i 23} \triangleq-N_{2 i}-S_{i 2}+S_{i 3}^{T}-M_{i 3}^{T} \\
& \Lambda_{i 33} \triangleq-R-N_{i 3}-S_{i 3}-N_{i 3}^{T}-S_{i 3}^{T}
\end{aligned}
$$

Proof: Consider the unforced system (1) and construct a stochastic Lyapunov functional as

$$
V\left(x_{k}, r_{k}\right)=\sum_{s=1}^{5} V_{s}\left(x_{k}, r_{k}\right),
$$

where $\forall r_{k}=i \in \mathcal{I}$

$$
\begin{aligned}
& V_{1}\left(x_{k}, r_{k}\right) \triangleq x_{k}^{T} P_{i} x_{k} \\
& V_{2}\left(x_{k}, r_{k}\right) \triangleq \sum_{l=k-d_{i}}^{k-1} x^{T}(l) Q x(l) \\
& V_{3}\left(x_{k}, r_{k}\right) \triangleq \sum_{\theta=-d_{M}+1} \sum_{l=k+\theta}^{-d_{m}} x^{T}(l) Q x(l) \\
& V_{4}\left(x_{k}, r_{k}\right) \triangleq \sum_{l=k-d_{M}}^{k-1} x^{T}(l) R x(l) \\
& V_{5}\left(x_{k}, r_{k}\right) \triangleq \sum_{\theta=-d_{M}} \sum_{l=k+\theta}^{k-1} y^{T}(l)\left(Z_{1}+Z_{2}\right) y(l)
\end{aligned}
$$


with $y(l) \triangleq x(l+1)-x(l)$ and $P_{i}, Q, R, Z_{1}, Z_{2}$ satisfying (5). Then, for $r_{k}=i, r_{k+1}=j$, we denote $\Delta V\left(x_{k}, r_{k}\right)=\sum_{s=1}^{5} \Delta V_{s}$, where

$\Delta V_{1} \triangleq E\left[V_{1}\left(x_{k+1}, r_{k+1} \mid x_{k}, r_{k}\right)-V_{1}\left(x_{k}, r_{k}\right)\right]$

$$
\begin{aligned}
= & x_{k+1}^{T} \sum_{j \in \mathcal{I}} \pi_{i j} P_{j} \tilde{x}_{k+1}-x_{k}^{T} P_{i} x_{k} \\
= & x_{k}^{T}\left(A_{i}^{T} \overline{\mathcal{P}}_{i} A_{i}-P_{i}\right) x_{k}+2 x_{k}^{T} A_{i}^{T} \overline{\mathcal{P}}_{i} A_{d i} x_{k-d(k)} \\
& +x_{k}^{T}-d(k) A_{d i}^{T} \overline{\mathcal{P}}_{i} A_{d i} x_{k-d(k)}
\end{aligned}
$$

$\Delta V_{2} \triangleq E\left[V_{2}\left(x_{k+1}, r_{k+1} \mid x_{k}, r_{k}\right)-V_{2}\left(x_{k}, r_{k}\right)\right]$

$$
\begin{aligned}
= & \left(\sum_{l=k+1-d_{k+1}}^{k}-\sum_{l=k-d_{k}}^{k-1}\right) x^{T}(l) Q x(l) \\
= & x^{T}(k) Q x(k)-x^{T}\left(k-d_{k}\right) Q x\left(k-d_{k}\right) \\
& +\sum_{l=k+1-d_{k+1}}^{k-d_{k}} x^{T}(l) Q x(l) \\
\leq & x^{T}(k) Q x(k)-x^{T}\left(k-d_{k}\right) Q x\left(k-d_{k}\right) \\
& +\sum_{l=k-d_{M}+1}^{k-d_{m}} x^{T}(l) Q x(l)
\end{aligned}
$$

$\Delta V_{3} \triangleq E\left[V_{3}\left(x_{k+1}, r_{k+1} \mid x_{k}, r_{k}\right)-V_{3}\left(x_{k}, r_{k}\right)\right]$

$$
\begin{aligned}
& =\sum_{\theta=-d_{M}+1}^{-d_{m}}\left(\sum_{l=k+\theta+1}^{k}-\sum_{l=k+\theta}^{k-1}\right) x^{T}(l) Q x(l) \\
& =\left(d_{M}-d_{m}\right) x^{T}(k) Q x(k)-\sum_{l=k-d_{M}+1}^{k-d_{m}} x^{T}(l) Q x(l)
\end{aligned}
$$

$\Delta V_{4} \triangleq E\left[V_{4}\left(x_{k+1}, r_{k+1} \mid x_{k}, r_{k}\right)-V_{4}\left(x_{k}, r_{k}\right)\right]$

$$
\begin{aligned}
& =\left(\sum_{l=k+1-d_{M}}^{k}-\sum_{l=k-d_{M}}^{k-1}\right) x^{T}(l) R x(l) \\
& =x^{T}(k) R x(k)-x^{T}\left(k-d_{M}\right) R x\left(k-d_{M}\right)
\end{aligned}
$$

$$
\begin{aligned}
\Delta V_{5} \triangleq & \sum_{\theta=-d_{M}}^{-1}\left[\sum_{l=k+\theta+1}^{k} y^{T}(l)\left(Z_{1}+Z_{2}\right) y(l)\right. \\
& \left.-\sum_{l=k+\theta}^{k-1} y^{T}(l)\left(Z_{1}+Z_{2}\right) y(l)\right] \\
= & \sum_{\theta=-d_{M}}^{-1}\left(\sum_{l=k+\theta+1}^{k}-\sum_{l=k+\theta}^{k-1}\right) y^{T}(l)\left(Z_{1}+Z_{2}\right) y(l) \\
= & \sum_{\theta=-d_{M}}^{-1}\left[y^{T}(k)\left(Z_{1}+Z_{2}\right) y(k)\right. \\
= & d_{M} y^{T}(k)\left(Z_{1}+Z_{2}\right) y(k)-\sum_{l=k-d_{k}}^{k-1} y^{T}(l) Z_{1} y(l) \\
& -\sum_{l=k-d_{M}}^{k-1} y^{T}(l) Z_{1} y(l)-\sum_{l=k-d_{M}}^{k-1} y^{T}(l) Z_{2} y(l)
\end{aligned}
$$

then we have

$$
\begin{aligned}
& \Delta V\left(x_{k}, r_{k}\right) \\
& \leq x_{k}^{T}\left(A_{i}^{T} \overline{\mathcal{P}}_{i} A_{i}-P_{i}\right) x_{k}+2 x_{k}^{T} A_{i}^{T} \overline{\mathcal{P}}_{i} A_{d i} x_{k-d_{k}} \\
& \quad+x_{k-d_{k}}^{T} A_{d i}^{T} \overline{\mathcal{P}}_{i} A_{d i} x_{k-d_{k}}-x^{T}\left(k-d_{k}\right) Q x\left(k-d_{k}\right) \\
& \quad+\left(d_{M}-d_{m}+1\right) x^{T}(k) Q x(k)+x^{T}(k) R x(k)
\end{aligned}
$$

$$
\begin{aligned}
& -x^{T}\left(k-d_{M}\right) R x\left(k-d_{M}\right) \\
& +d_{M}\left[\left(A_{i}-I\right) x(k)+A_{d i} x\left(k-d_{k}\right)\right]^{T} \\
& \times\left(Z_{1}+Z_{2}\right)\left[\left(A_{i}-I\right) x(k)+A_{d i} x\left(k-d_{k}\right)\right] \\
& -\sum_{l=k-d_{k}}^{k-1} y^{T}(l) Z_{1} y(l)-\sum_{l=k-d_{M}}^{k-d_{k}-1} y^{T}(l) Z_{1} y(l) \\
& -\sum_{l=k-d_{M}}^{k-1} y^{T}(l) Z_{2} y(l) \\
& +2 \zeta^{T}(k) M_{i}\left[x(k)-x\left(k-d_{k}\right)-\sum_{l=k-d_{k}}^{k-1} y(l)\right] \\
& +2 \zeta^{T}(k) S_{i}\left[x\left(k-d_{k}\right)-x\left(k-d_{M}\right)-\sum_{l=k-d_{M}}^{k-d_{k}-1} y(l)\right] \\
& +2 \zeta^{T}(k) N_{i}\left[x(k)-x\left(k-d_{M}\right)-\sum_{l=k-d_{M}}^{k-1} y(l)\right]
\end{aligned}
$$

Therefore, we obtain

$$
\begin{aligned}
\Delta V\left(x_{k}, r_{k}\right) \leq & \zeta^{T}(k)\left[\Omega_{i}+\Theta_{i}+d_{M} M_{i} Z_{1}^{-1} M_{i}^{T}+\left(d_{M}-d_{m}\right)\right. \\
& \left.\times S_{i} Z_{1}^{-1} S_{i}^{T}+d_{M} N_{i} Z_{2}^{-1} N_{i}^{T}\right] \zeta(k) \\
& -\sum_{l=k-d_{k}}^{k-1}\left[\zeta^{T}(k) M_{i}+y^{T}(l) Z_{1}\right] Z_{1}^{-1} \\
& \times\left[\zeta^{T}(k) M_{i}+y^{T}(l) Z_{1}\right]^{T} \\
& -\sum_{l=k-d_{M}}^{k-d_{k}-1}\left[\zeta^{T}(k) S_{i}+y^{T}(l) Z_{1}\right] Z_{1}^{-1} \\
& \times\left[\zeta^{T}(k) S_{i}+y^{T}(l) Z_{1}\right]^{T} \\
& -\sum_{l=k-d_{M}}^{k-1}\left[\zeta^{T}(k) N_{i}+y^{T}(l) Z_{2}\right] Z_{2}^{-1} \\
& \times\left[\zeta^{T}(k) N_{i}+y^{T}(l) Z_{2}\right]^{T}
\end{aligned}
$$

where

$$
\begin{aligned}
\zeta(k) \triangleq & {\left[\begin{array}{ccc}
x_{k}^{T} & x^{T}\left(k-d_{k}\right) & x^{T}\left(k-d_{M}\right)
\end{array}\right]^{T} \text { and } } \\
\Omega_{i} \triangleq & {\left[\begin{array}{ccc}
\Omega_{i 1} & \Omega_{i 2} & 0 \\
* & \Omega_{i 3} & 0 \\
* & * & -R
\end{array}\right] } \\
\Theta_{i} \triangleq & {\left[\begin{array}{lll}
M_{i}+N_{i} & S_{i}-M_{i} & -N_{i}-S_{i}
\end{array}\right] } \\
& +\left[\begin{array}{lll}
M_{i}+N_{i} & S_{i}-M_{i} & -N_{i}-S_{i}
\end{array}\right]^{T}
\end{aligned}
$$

with

$$
\begin{aligned}
\Omega_{i 1} \triangleq & {\left[\begin{array}{l}
A_{i}^{T} \overline{\mathcal{P}}_{i} A_{i}-P_{i}+\left(d_{M}-d_{m}+1\right) Q+R \\
\quad+d_{M}\left(A_{i}-I\right)^{T}\left(Z_{1}+Z_{2}\right)\left(A_{i}-I\right)
\end{array}\right] } \\
\Omega_{i 2} \triangleq & d_{M}\left(A_{i}-I\right)^{T}\left(Z_{1}+Z_{2}\right) A_{d i}+A_{i}^{T} \overline{\mathcal{P}}_{i} A_{d i} \\
\Omega_{i 3} \triangleq & d_{M} A_{d i}\left(Z_{1}+Z_{2}\right) A_{d i}^{T}+A_{d i}^{T} \overline{\mathcal{P}}_{i} A_{d i}-Q \\
M_{i} \triangleq & {\left[\begin{array}{lll}
M_{i 1}^{T} & M_{i 2}^{T} & M_{i 3}^{T}
\end{array}\right]^{T} } \\
N_{i} \triangleq & {\left[\begin{array}{lll}
N_{i 1}^{T} & N_{i 2}^{T} & N_{i 3}^{T}
\end{array}\right]^{T} } \\
S_{i} \triangleq & {\left[\begin{array}{lll}
S_{i 1}^{T} & S_{i 2}^{T} & S_{i 3}^{T}
\end{array}\right]^{T} }
\end{aligned}
$$


Then, since both $Z_{1}>0$ and $Z_{2}>0$, the last three terms are nonpositive in (6). By Schur complement, (5) guarantees $\Omega_{i}+\Theta_{i}+d_{M} M_{i} Z_{1}^{-1} M_{i}^{T}+\left(d_{M}-d_{m}\right) S_{i} Z_{1}^{-1} S_{i}^{T}+d_{M} N_{i} Z_{2}^{-1} N_{i}^{T}<$ 0 . Therefore, we have $\Delta V\left(x_{k}, r_{k}\right)<-\delta\|x(k)\|^{2}$ for a sufficiently small $\delta>0$ and $x(k) \neq 0$. Following a similar line in the proof of Theorem 1 in [12], it can be shown that $E\left\{\sum_{k=0}^{\infty}\left\|x_{k}\right\|^{2}\right\}<\infty$, that is, the system is stochastically stable.

Now, the following theorem presents a sufficient condition for the stochastic stability of system (1) with partially known transition probabilities (2).

Theorem 1: Consider the unforced system (1) with partially known transition probabilities (2). The corresponding system is stochastically stable if there exist matrices $P_{i}>0, i \in \mathcal{I}, Q>0, R>0, Z_{v}>0$, $v=1,2, M_{i v}, N_{i v}, S_{i v}, v=1,2,3, \forall i \in \mathcal{I}$ such that

$$
\left[\begin{array}{cccc}
-\Upsilon_{j} & 0 & 0 & \Psi_{i 5} \\
* & -Z_{2} & 0 & \Psi_{i 2} \\
* & * & -Z_{1} & \Psi_{i 3} \\
* & * & * & \Psi_{i 4}
\end{array}\right]<0
$$

where $\Psi_{i 5} \triangleq\left[\Upsilon_{j} A_{i} \Upsilon_{j} A_{d i} \quad \begin{array}{llll}0 & 0 & 0 & 0\end{array}\right], \Psi_{i v}, v=2,3,4$ are defined in Proposition 1 and if $\pi_{\mathcal{K}}^{i}=0, \Upsilon_{j} \triangleq P_{j}$, otherwise,

$$
\left\{\begin{array}{l}
\Upsilon_{j} \triangleq \frac{1}{\pi_{\mathcal{K}}^{i}} \mathcal{P}_{\mathcal{K}}^{i} \\
\Upsilon_{j} \triangleq P_{j}, \forall j \in \mathcal{I}_{\mathcal{U} \mathcal{K}}^{i}
\end{array}\right.
$$

with $\mathcal{P}_{\mathcal{K}}^{i} \triangleq \sum_{j \in \mathcal{I}_{\mathcal{K}}^{i}} \pi_{i j} P_{j}$.

Proof: First of all, we know that the unforced system (1) is stochastically stable under the completely known transition probabilities (2) if (5) holds. Note that (5) can be rewritten as

$$
\Xi_{i} \triangleq\left[\begin{array}{cc}
-\mathcal{P}_{\mathcal{K}}^{i} & \mathcal{P}_{\mathcal{K}}^{i} \Xi_{i 1} \\
* & \pi_{\mathcal{K}}^{i} \Xi_{i 2}
\end{array}\right]+\sum_{j \in \mathcal{I}_{\mathcal{U} \mathcal{K}}^{i}} \pi_{i j}\left[\begin{array}{cc}
-P_{j} & P_{j} \Xi_{i 1} \\
* & \Xi_{i 2}
\end{array}\right]
$$

where

$$
\begin{aligned}
& \Xi_{i 1} \triangleq\left[\begin{array}{llllllll}
0 & 0 & A_{i} & A_{d i} & 0 & 0 & 0 & 0
\end{array}\right] \text { and } \\
& \Xi_{i 2} \triangleq\left[\begin{array}{ccc}
-Z_{2} & 0 & \Psi_{i 2} \\
* & -Z_{1} & \Psi_{i 3} \\
* & * & \Psi_{i 4}
\end{array}\right]
\end{aligned}
$$

Therefore, if one has

$$
\begin{aligned}
& {\left[\begin{array}{cc}
-\mathcal{P}_{\mathcal{K}}^{i} & \mathcal{P}_{\mathcal{K}}^{i} \Xi_{i 1} \\
* & \pi_{\mathcal{K}}^{i} \Xi_{i 2}
\end{array}\right]<0,} \\
& {\left[\begin{array}{cc}
-P_{j} & P_{j} \Xi_{i 1} \\
* & \Xi_{i 2}
\end{array}\right]<0, \forall j \in \mathcal{I}_{\mathcal{U} \mathcal{K}}^{i},}
\end{aligned}
$$

then we have $\Xi_{i}<0$, hence the system is stochastically stable under partially known transition probabilities, which is concluded from the obvious fact that no knowledge on $\pi_{i j}, \forall j \in \mathcal{I}_{\mathcal{U} \mathcal{K}}^{i}$ is required in (8) and (9). Thus, for $\pi_{\mathcal{K}}^{i} \neq 0$ and $\pi_{\mathcal{K}}^{i}=0$, respectively, one can readily obtain (7), since if $\pi_{\mathcal{K}}^{i}=0$, the conditions (8), (9) will reduce to (9). This completes the proof.

Now let us consider the stabilizing controller design. From the above development, it can be seen that the system with completely known transition probabilities is just a special case of our considered systems.
In what follows, we will give a stabilization condition of the system with partially known transition probabilities as generalized results.

Theorem 2: Consider system (1) with partially known transition probabilities (2). There exists a controller (4) such that the resulting closed-loop system is stochastically stable if there exist matrices $P_{i}>$ $0, X_{i}, i \in \mathcal{I}, Q>0, R>0, Z_{v}>0, U_{v}>0, v=1,2, M_{i v}, N_{i v}$, $S_{i v}, v=1,2,3, \forall i \in \mathcal{I}$ and $K_{i}$ such that

$$
\begin{gathered}
{\left[\begin{array}{cccc}
-\hat{\Upsilon}_{j} & 0 & 0 & \Psi_{i 6} \\
* & -U_{2} & 0 & \Psi_{i 7} \\
* & * & -U_{1} & \Psi_{i 7} \\
* & * & * & \Psi_{i 4}
\end{array}\right]<0} \\
P_{i} X_{i}=I, \quad Z_{1} U_{1}=I, \quad Z_{2} U_{2}=I
\end{gathered}
$$

where

$$
\begin{aligned}
& \Psi_{i 6} \triangleq\left[\begin{array}{llllll}
\mathcal{L}_{j}\left(A_{i}+B_{i} K_{i}\right) & \mathcal{L}_{j} A_{d i} & 0 & 0 & 0 & 0
\end{array}\right] \\
& \Psi_{i 7} \triangleq\left[\begin{array}{llllll}
\sqrt{d_{M}}\left(A_{i}+B_{i} K_{i}-I\right) & \sqrt{d_{M}} A_{d i} & 0 & 0 & 0 & 0
\end{array}\right],
\end{aligned}
$$

$\Psi_{i 4}$ is defined in Proposition 1 and if $\pi_{\mathcal{K}}^{i}=0, \hat{\Upsilon}_{j} \triangleq X_{j}$ and $\mathcal{L}_{j} \triangleq I$, otherwise,

$$
\left\{\begin{array}{l}
\hat{\Upsilon}_{j} \triangleq \pi_{\mathcal{K}}^{i} \operatorname{diag}\left\{X_{\mathcal{K}_{1}^{i}}, \ldots, X_{\mathcal{K}_{m}^{i}}\right\} \\
\mathcal{L}_{j} \triangleq\left[\sqrt{\pi_{i \mathcal{K}_{1}^{i}}} I, \ldots, \sqrt{\pi_{i \mathcal{K}_{m}^{i}}} I\right]^{T} \\
\hat{\Upsilon}_{j} \triangleq X_{j}, \quad \mathcal{L}_{j} \triangleq I, \quad \forall j \in \mathcal{I}_{\mathcal{U}}^{i} \mathcal{K}
\end{array}\right.
$$

Moreover, if (10), (11) have solutions, the controller gain is given by $K_{i}$.

Proof: By Schur complement, (7) is equivalent to (for $\pi_{\mathcal{K}}^{i} \neq 0$ )

$$
\begin{gathered}
\Xi_{i 3}+\Xi_{i 4}<0, \\
{\left[\begin{array}{cc}
-P_{j}^{-1} & \Xi_{i 1} \\
* & \Xi_{i 3}
\end{array}\right]<0, \quad \forall j \in \mathcal{I}_{\mathcal{U K}}^{i}}
\end{gathered}
$$

where $\Xi_{i 1}$ is defined in Theorem 1 and

$$
\begin{aligned}
& \Xi_{i 3} \triangleq\left[\begin{array}{ccccc}
-Z_{2}^{-1} & 0 & \Psi_{i 8} & -Z_{1}^{-1} & \Psi_{i 8} \\
* & * & \Psi_{i 4} & &
\end{array}\right] \\
& \Xi_{i 4} \triangleq\left[\begin{array}{cccccccc}
0 & 0 & 0 & 0 & 0 & 0 & 0 & 0 \\
* & 0 & 0 & 0 & 0 & 0 & 0 & 0 \\
* & * & \frac{1}{\pi_{\mathcal{K}}^{i}} A_{i}^{T} \mathcal{P}_{\mathcal{K}}^{i} A_{i} & \frac{1}{\pi_{\mathcal{K}}^{i}} A_{i}^{T} \mathcal{P}_{\mathcal{K}}^{i} A_{d i} & 0 & 0 & 0 & 0 \\
* & * & * & \frac{1}{\pi_{\mathcal{K}}^{i}} A_{d i}^{T} \mathcal{P}_{\mathcal{K}}^{i} A_{d i} & 0 & 0 & 0 & 0 \\
* & * & * & * & 0 & 0 & 0 & 0 \\
* & * & * & * & * & 0 & 0 & 0 \\
* & * & * & * & * & * & 0 & 0 \\
* & * & * & * & * & * & * & 0
\end{array}\right]
\end{aligned}
$$

with

$$
\Psi_{i 8} \triangleq\left[\begin{array}{llllll}
\sqrt{d_{M}}\left(A_{i}-I\right) & \sqrt{d_{M}} A_{d i} & 0 & 0 & 0 & 0
\end{array}\right]
$$

Bearing the notations $\mathcal{I}_{\mathcal{K}}^{i}=\left\{\mathcal{K}_{1}^{i}, \ldots, \mathcal{K}_{m}^{i}\right\}$ and $\mathcal{P}_{\mathcal{K}}^{i}=\sum_{j \in \mathcal{I}_{\mathcal{K}}^{i}} \pi_{i j} P_{j}$ in mind and by Schur complement again ( $\mathcal{K}_{m}^{i}$ times), we have (13) is equivalent to

$$
\left[\begin{array}{cc}
\Xi_{i 5} & \Xi_{i 6} \\
* & \Xi_{i 3}
\end{array}\right]<0
$$


where

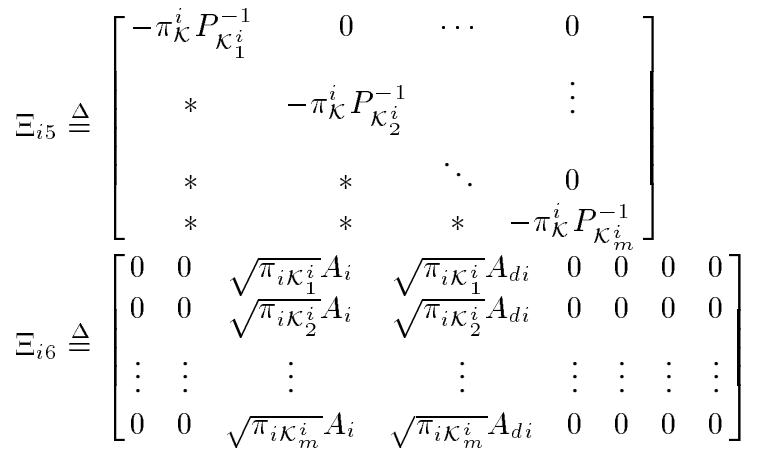

Note that if $\pi_{\mathcal{K}}^{i}=0$, (7) will be just equivalent to (14). Then, consider the system with the control input (4), replace $A_{i}$ in (14) and (15) by $A_{i}+B_{i} K_{i}$, and set $X_{i} \triangleq P_{i}^{-1}, U_{v}=Z_{v}^{-1}, v=1,2$, and $\hat{\Upsilon}_{j}$ and $\mathcal{L}_{j}$ as shown in (12), we can readily obtain (10) and (11). This completes the proof.

Remark 2: It should be noted that the conditions stated in Theorem 2 are actually a set of LMIs with some matrix inverse constraints. Although they are nonconvex, which prevents us from solving them using the existing convex optimization tool, there exist approaches to solve them such as the cone complementary linearization (CCL) algorithm developed in [16], which has been demonstrated to be efficient. Therefore, it is suggested here to use CCL algorithm to calculate the controller gains from Theorem 2. Also, one can find a suboptimal $d_{M}$ for given $d_{m}$ by using the bisection technique.

Remark 3: There is a monotonicity in the reduction of conservatism of the condition in Theorem 1 as the number of known probabilities increases. In other words, the more known elements there are in the transition probability matrix, the lower the conservatism of the condition will be. If all the transition probabilities are unknown to the designers (i.e. $\pi_{\mathcal{K}}^{i}=0$ ), the corresponding system can be viewed as a switched linear system under arbitrary switching. Therefore, the conditions obtained in Proposition 1 and Theorem 1 will thereby cover the results for arbitrary switched linear systems with time-varying delays if one derives them based on delay-range-dependent technique and switched Lyapunov function approach [17]. Naturally, without the information of transition probabilities for designers, the achieved system performance (say, the maximal admissible delays) might be conservative, which would be further demonstrated in the Section IV.

\section{NUMERICAL EXAMPLE}

Consider the MJLS (1) with four operation modes and the following data:

$$
\begin{aligned}
A_{1} & =\left[\begin{array}{cc}
-1.16 & 0.54 \\
0.23 & -0.92
\end{array}\right], \quad A_{2}=\left[\begin{array}{ll}
0.92 & 0.54 \\
0.23 & 0.92
\end{array}\right] \\
A_{3} & =\left[\begin{array}{cc}
0.77 & 0.54 \\
0.23 & -0.92
\end{array}\right], \quad A_{4}=\left[\begin{array}{cc}
-1.16 & 0.54 \\
0.23 & 0.92
\end{array}\right] \\
A_{d 1} & =\left[\begin{array}{cc}
-0.02 & 0.12 \\
0.07 & -0.14
\end{array}\right], \quad A_{d 2}=\left[\begin{array}{cc}
0.02 & 0.12 \\
0.07 & 0.02
\end{array}\right] \\
A_{d 3} & =\left[\begin{array}{cc}
-0.02 & 0.12 \\
0.07 & 0.02
\end{array}\right], \quad A_{d 4}=\left[\begin{array}{cc}
0.02 & 0.12 \\
0.07 & -0.14
\end{array}\right] \\
B_{1} & =\left[\begin{array}{c}
-3.0 \\
1.6
\end{array}\right], \quad B_{2}=\left[\begin{array}{c}
0.5 \\
-0.08
\end{array}\right] \\
B_{3} & =\left[\begin{array}{c}
0.5 \\
0.2
\end{array}\right], \quad B_{4}=\left[\begin{array}{c}
-0.7 \\
0.2
\end{array}\right]
\end{aligned}
$$

and four cases for the transition probabilities matrix are given in Table I.

Our purpose here is to check the stability of the above system without control and design a stabilizing controller of the form (4) for the four
TABLE I

DiFFERENT TRANSITION PROBABILITY MATRICES

\begin{tabular}{c|c|c|c|c|}
\hline \hline \multicolumn{5}{c}{ Completely known } \\
\hline & 1 & 2 & 3 & 4 \\
\hline 1 & 0.4 & 0.6 & 0 & 0 \\
\hline 2 & 0.2 & 0.6 & 0.19 & 0.01 \\
\hline 3 & 0.2 & 0.3 & 0.4 & 0.1 \\
\hline 4 & 0.3 & 0.3 & 0.3 & 0.1 \\
\hline
\end{tabular}

Partially known (Case I)

\begin{tabular}{|c|c|c|c|c|}
\hline & 1 & 2 & 3 & 4 \\
\hline 1 & 0.4 & 0.6 & 0 & 0 \\
\hline 2 & 0.2 & 0.6 & 0.19 & 0.01 \\
\hline 3 & $?$ & 0.3 & $?$ & 0.1 \\
\hline 4 & 0.3 & $?$ & 0.3 & $?$ \\
\hline
\end{tabular}

Partially known (Case II)

\begin{tabular}{|c|c|c|c|c|}
\hline & 1 & 2 & 3 & 4 \\
\hline 1 & $?$ & 0.6 & 0 & $?$ \\
\hline 2 & 0.2 & 0.6 & 0.19 & 0.01 \\
\hline 3 & $?$ & 0.3 & $?$ & 0.1 \\
\hline 4 & 0.3 & $?$ & 0.3 & $?$ \\
\hline
\end{tabular}

Completely unknown

\begin{tabular}{|l|l|l|l|l|}
\hline & 1 & 2 & 3 & 4 \\
\hline 1 & $?$ & $?$ & $?$ & $?$ \\
\hline 2 & $?$ & $?$ & $?$ & $?$ \\
\hline 3 & $?$ & $?$ & $?$ & $?$ \\
\hline 4 & $?$ & $?$ & $?$ & $?$ \\
\hline
\end{tabular}

different cases of transition probabilities. First of all, given $d_{m}=$ $d_{M}=0$, the unforced system is unstable even if all the transition probabilities are known, which can be tested either by simulation or by stability criterion for MJLS without delays (the criterion is sufficient and necessary). It implies that the underlying system will be unstable for any time-varying delay starting from $d_{m}=0$. Also, if $d_{m}=1$, one can check by simulation that the unforced system is unstable even for the smallest range $1 \leq d(k) \leq 1$. Then, assume $d_{m}=1$ and solve (10), (11) in Theorem 2 using the CCL algorithm combined with bisection technique, the stabilizing controller gains and the delay ranges for the different cases can be computed, respectively, as shown in Table II.

It is easily seen from Table II that the more transition probabilities knowledge we have, the larger the delay range can be obtained for ensuring stability. This shows the tradeoff between the cost of obtaining transition probabilities and the system performance (the maximal admissible delay ranges in this example).

Furthermore, applying the obtained controllers, giving random time-varying delays within the corresponding ranges and giving system modes evolutions, one can test and observe the state response of the resulting closed-loop system. Now, assign fixed values to the unknown elements in the partially known transition probability matrix (Case I) in Table I, and consider two possible transition probability matrices in practice as shown in Table III.

Then, giving two different series of delays $d_{1}(k)$ and $d_{2}(k)$ and two possible modes variations $r_{1}(k)$ and $r_{2}(k)$ generated based on the two matrices in Table III (those elements in brackets are treated as unknown in the controller design process), respectively, we get the state response using the controller obtained for the system with partially known transition probabilities (Case I) as shown in Figs. 1 and 2 for the given initial condition $x(s)=[0.5-0.3]^{T}, s=-7,-6, \ldots, 0$. It is obvious that the designed controller is feasible and ensures the stability of the closed-loop system despite the partially unknown transition probabilities and the time-varying delays.

\section{CONCLUSION}

The stability analysis and stabilization problem for a class of discrete-time Markov jump linear system (MJLS) with partially known 
TABLE II

CONTROller Gains AND Delay RANGES For DifFERENT CASES

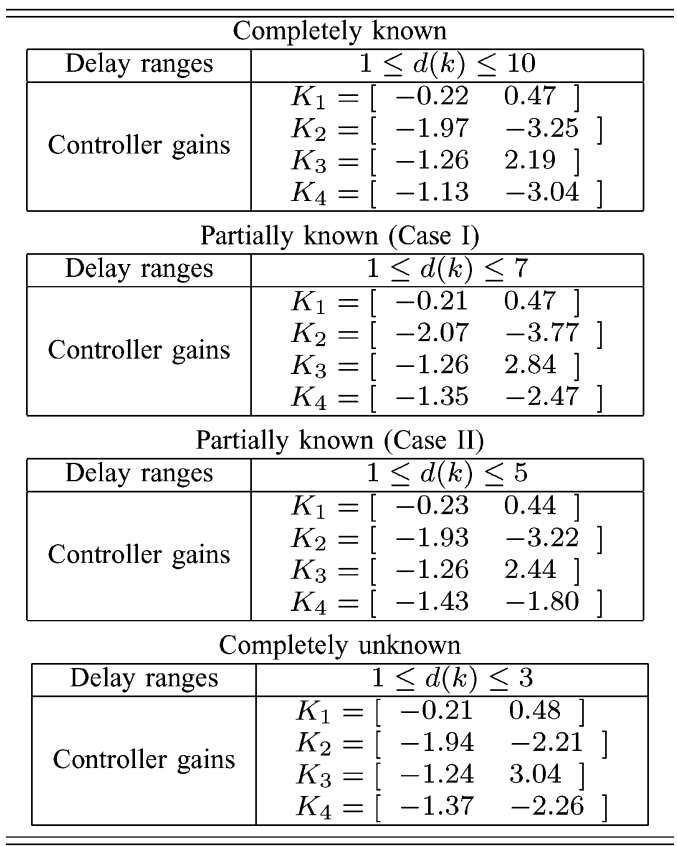

TABLE III

The Possible Transition Probability Matrices for CaSe I in TABle I

\begin{tabular}{|c|c|c|c|c|}
\hline \multicolumn{5}{|c|}{ Matrix 1} \\
\hline & 1 & 2 & 3 & 4 \\
\hline 1 & 0.4 & 0.6 & 0 & 0 \\
\hline 2 & 0.2 & 0.6 & 0.19 & 0.01 \\
\hline 3 & $(0.2)$ & 0.3 & $(0.4)$ & 0.1 \\
\hline 4 & 0.3 & $(0.3)$ & 0.3 & $(0.1)$ \\
\hline \multicolumn{5}{|c|}{ Matrix 2} \\
\hline & 1 & 2 & 3 & 4 \\
\hline 1 & 0.4 & 0.6 & 0 & 0 \\
\hline 2 & 0.2 & 0.6 & 0.19 & 0.01 \\
\hline 3 & $(0.5)$ & 0.3 & $(0.1)$ & 0.1 \\
\hline 4 & 0.3 & $(0.2)$ & 0.3 & $(0.2)$ \\
\hline
\end{tabular}

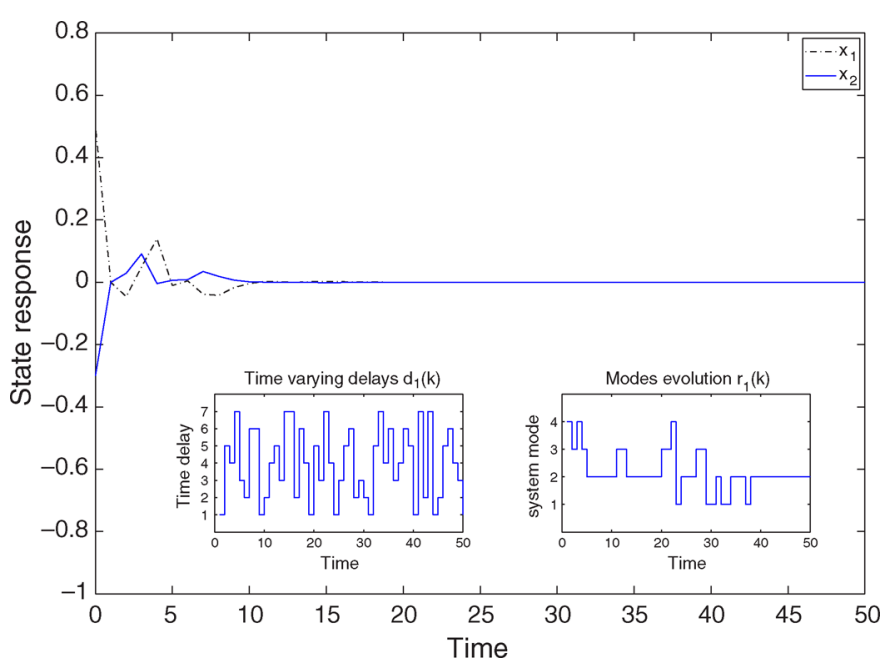

Fig. 1. State response of the closed-loop system for $d_{1}(k)$ and $r_{1}(k)$.

transition probabilities and time-varying delays are investigated in this note. The considered time-varying delays and partially known transi-

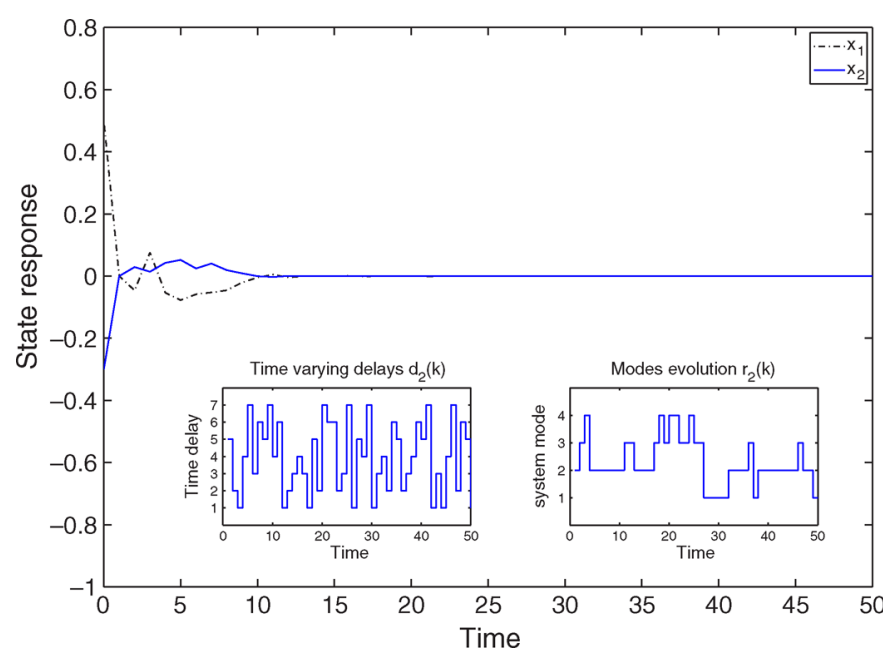

Fig. 2. State response of the closed-loop system for $d_{2}(k)$ and $r_{2}(k)$.

tion probabilities are more general and practicable in MJLS. A tradeoff can be observed between the complexity of obtaining all the transition probabilities and the maximal admissible delay range for ensuring stability. It is worth expecting that the methods and ideas behind the note could be applied to solve other issues on the underlying systems, such as $H_{\infty}$ control, filtering, etc.

\section{REFERENCES}

[1] E. K. Boukas and Z. K. Liu, Deterministic and Stochastic Time-Dealy Systems. Berlin, Germany: Birkhauser, 2002.

[2] H. Gao, J. Lam, C. Wang, and Y. Wang, "Delay-dependentoutputfeedback stabilisation of discrete-time systems with time-varying statedelay," IET Control Theory Appl., vol. 151, no. 6, pp. 691-698, 2004.

[3] P. Park, "A delay-dependent stability criterion for systems withuncertain time-invariant delays," IEEE Trans. Automat. Control, vol. 44, no. 4, pp. 876-877, Apr. 1999.

[4] L. Zhang, P. Shi, and E. Boukas, “ $H_{\infty}$ output-feedback control for switched linear discrete-time systems withtime-varying delays," Int. J. Control, vol. 80, no. 8, pp. 1354-1365, 2007.

[5] L. Zhang, P. Shi, E. Boukas, and C. Wang, "Robust $l_{2}-l_{\infty}$ filtering for switchedlinear discrete time-delay systems with polytopic uncertainties," IET Control Theory Appl., vol. 1, no. 3, pp. 722-730, 2007.

[6] H. Gao and T. Chen, "New results on stability of discrete-timesystems with time-varying state delay," IEEE Trans. Automat. Control, vol. 52, no. 2, pp. 328-334, Feb. 2007.

[7] Y. He, Q. G. Wang, C. Lin, and M. Wu, "Delay-range-dependent stability for systems with time-varying delay," Automatica, vol. 43, no. 2 , pp. 371-376, 2007.

[8] E. K. Boukas, Stochastic Switching Systems: Analysis and Design. Berlin, Germany: Birkhauser, 2005.

[9] O. L. V. Costa, M. D. Fragoso, and R. P. Marques, Discrete-Time Markovian Jump Linear Systems. London, U.K.: Springer-Verlag, 2005.

[10] D. P. de Farias, J. C. Geromel, J. B. R. do Val, and O. L. V. Costa, "Output feedback control of markov jump linear systems incontinuoustime," IEEE Trans. Automat. Control, vol. 45, no. 5, pp. 944-949, May 2000.

[11] L. Zhang and E. K. Boukas, "Stability and stabilization of markovian jump linear systems with partly unknown transition probabilities," in Proc. 17th IFAC World Congress, Seoul, Korea, 2008, accepted for publication.

[12] E. K. Boukas and Z. K. Liu, "Robust $H_{\infty}$ control of discrete-time Markovian jump linear systems with mode-dependent time-delays," IEEE Trans. Automat. Control, vol. 46, no. 12, pp. 1918-1924, Dec. 2001.

[13] C. E. De Souza, A. Trofino, and K. A. Barbosa, "Mode-independent $H_{\infty}$ filters for Markovian jump linearsystems," IEEE Trans. Automat. Control, vol. 51, no. 11, pp. 1837-1841, Nov. 2006. 
[14] M. Karan, P. Shi, and C. Y. Kaya, "Transition probability bounds for the stochastic stability robustness of continuous- and discrete-time Markovian jump linear systems," Automatica, vol. 42, no. 12, pp. 2159-2168, 2006.

[15] J. Xiong, J. Lam, H. Gao, and D. Ho, "On robust stabilization ofmarkovian jump systems with uncertain switching probabilities," Automatica, vol. 41, no. 5, pp. 897-903, 2005.

[16] L. El Ghaoui, F. Oustry, and M. Ait Rami, "A cone complementarity linearization algorithm for static output-feedback and relatedproblems," IEEE Trans. Automat. Control, vol. 42, no. 8, pp. 1171-1176, Aug. 1997.

[17] J. Daafouz, P. Riedinger, and C. Iung, "Stability analysis andcontrol synthesis for switched systems: A switched Lyapunov functionapproach," IEEE Trans. Automat. Control, vol. 47, no. 11, pp. 1883-1887, Nov. 2002.

\section{Corrections to "Asynchronous Consensus in Continuous-Time Multi-Agent Systems With Switching Topology and Time-Varying Delays"'}

In the above paper [1], the location that was given for Inner Mongolia University within author Feng Xiao's biography is incorrect. The correct version of the biographical listing is shown here. Also, all of the official received and revised dates were not shown in the first footnote. The paper's received date was December 7, 2005. The revised dates were February 8, 2007 and June 21, 2007.

\section{REFERENCES}

[1] F. Xiao and L. Wang, "Asynchronous Consensus in Continuous-Time Multi-Agent Systems With Switching Topology and Time-Varying Delays," IEEE Trans. Automat. Control, vol. 53, no. 8, pp. 1804-1816, Sep. 2008.

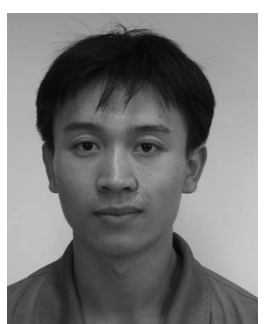

Feng Xiao received the B.S. and M.S. degrees in mathematics from Inner Mongolia University, Hohhot, China, in 2001 and 2004, respectively, and is currently pursuing the Ph.D. degree in complex systems at Peking University, Beijing, China.

His current research interests are in the fields of coordination of multi-agent systems, consensus problems, formation control, and swarm dynamics.

Digital Object Identifier 10.1109/TAC.2008.2007352 